\title{
Elaboración de un producto tipo 'pasta alimenticia' a partir de residuos de plátano hartón prefreído
}

\section{Food paste production from pre-fried harton plantain residues}

Fecha de recepción: 17 de septiembre de 2016

Sandra Patricia Chaparro-Acuña ${ }^{1}$

Fecha de aprobación: 28 de diciembre de 2016

\section{Resumen}

El propósito de este estudio fue aprovechar los recortes de plátano hartón prefreído, o subproducto de patacón (SPP), que las empresas producen en la elaboración de patacones y que representan, aproximadamente, un $30 \%$ de pérdidas mensuales. Dada la alta perecibilidad de estos residuos, se formuló un producto de buena aceptabilidad, con una vida útil más larga y que facilitara el manejo del subproducto. Se utilizaron tres harinas de trigo de diferente marca comercial, sémola de trigo y SPP en diferentes proporciones para establecer la mínima inclusión de harina en la formulación del producto tipo "pasta". Las pruebas de absorción y pérdida de agua determinaron que la mejor mezcla fue la que contenía $65 \%$ de residuos de plátano hartón prefreído y 35 \% de sémola de trigo. Las pruebas sensoriales con consumidores ayudaron a la selección de esta formulación. Los análisis de textura realizados mostraron mayor fracturabilidad y menor firmeza del producto tipo "pasta" con relación a una pasta patrón, pero durante la cocción se mantuvo estable y presentó buena consistencia.

Palabras clave: agroindustria; nuevos productos; plátano; plátano hartón; semolina.

\begin{abstract}
The goal of this study was to evaluate the use of harton plantain pre-fried residues (HPPR) that result from the patacon's manufacture, and that represent, in average, $30 \%$ of the mothly losses. Given the high perishability of these residues, a well-accepted product with longer endurance that facilitate the sub-product management was formulated. Three different brands of wheat flour and semolin were used to evaluate the lowest flour enclosed in the paste manufacture. The water absorption and loss tests showed that the best mixture contained $65 \%$ of HPPR and $35 \%$ of semolina. Sensory tests with consumers helped in the se-
\end{abstract}

1 M. Sc. Universidad Nacional de Colombia (Bogotá, D. C., Colombia). spchaparroa@unal.edu.co. 
lection of this formulation. The texture analysis showed that the dry pasta of HPPR is more fragile and less firm than a standard paste, but throughout cooking it remained stable and presented good consistency.

Keywords: agroindustry; harton; new products; plantain; semolina.

\section{Cómo citar este artículo:}

Chaparro-Acuña SP. Elaboración de un producto tipo 'pasta alimenticia' a partir de residuos de plátano hartón prefreído. Rev. Cien. Agri. 2017; 14(1): 47-56. 


\section{Introducción}

Los plátanos pertenecen a la familia de las Musáceas y comprenden los frutos procedentes de cualquier variedad del género Musa, híbridos triploides de Musa acuminata, Musa balbisiana, Musa cavendishii (plátanos comestibles cuando están crudos) y Musa paradisíaca (plátanos machos o para cocer) (1). El cultivo del plátano abarca rangos extremos de tolerancia, desde condiciones del bosque húmedo templado $\left(12-18{ }^{\circ} \mathrm{C} ; 4000-8000\right.$ $\mathrm{mm}$ de precipitación), hasta condiciones del Bosque muy seco Tropical (más de $24{ }^{\circ} \mathrm{C} ; 1000-1200$ $\mathrm{mm}$ de precipitación) (2 mid-vegetative and floral phases leading up to flowering (bunch emergence). Los plátanos y los bananos son cultivos de gran importancia en los países en vía de desarrollo; agricultores de Asia, África y América Latina generan su sustento de los cultivos de Musa como fuente de alimento, proporcionando a la población componentes nutricionales básicos en su dieta diaria como son: carbohidratos, fibra, proteínas, vitamina del complejo B y minerales como potasio, magnesio, fósforo, hierro y calcio (3). Las frutas que son inapropiadas para los estándares del mercado de exportación pueden ser procesadas en diferentes formas.

Colombia es el segundo productor mundial de plátanos, después de Uganda, con una producción de 2.925.000 toneladas por año, una participación de $9,2 \%$ y un rendimiento de 7,7 t/ha $(4,3)$. Dentro del contexto que conforman las especies vegetales que se cultivan en el país es indiscutible la importancia socioeconómica que tiene el cultivo del plátano; su valor se ha ido incrementando año tras año, por considerarse que no solo es uno de los componentes principales de la canasta familiar, sino también por su contribución en la generación de fuentes de trabajo y de divisas, al igual que como materia prima en industrias procesadoras de alimentos para consumo humano y animal (5).

Existen algunas técnicas para el procesamiento del plátano con el fin de obtener productos como patacones (freído directo, en profundidad o en la superficie), patacones congelados y plátano maduro congelado, pero, infortunadamente, se producen residuos, que la industria busca valorizar para aumentar beneficios y disminuir la contaminación que implique producción "verde" (6).

Los cereales son una especie vegetal perteneciente a la familia de las gramíneas; los más cultivados son el trigo, el maíz, el arroz, la cebada, la avena, el sorgo y el mijo. El trigo (Triticum) es, desde la prehistoria, el más importante de los cereales, debido a su adaptación a todo tipo de terreno y a diferentes climas. Actualmente se cultivan cerca de diez especies del género Triticum, pero solo dos presentan interés desde el punto de vista comercial: el Triticum vulgare y el Triticum durum (7). La harina de trigo es uno de los productos derivados del trigo más utilizados; su composición promedio es: agua $(12,8 \%)$, proteínas $(10,4 \%)$, carbohidratos $(75,1 \%)$ y lípidos $(0,5 \%)(8)$.

La pasta es un alimento consumido en la zona Mediterránea y muy apreciada por sus atributos de textura (9); su ingrediente principal es la semolina, de la cual deriva su coloración amarilla característica, debido a la presencia de pigmentos como los carotenoides (10), aunque también se ha elaborado con Triticum durum variedad morada para incrementar su contenido de antocianinas y carotenoides (11). La elaboración de la pasta involucra diferentes fases: mezclado de los ingredientes, formación de la masa, extrusión y secado (12); las principales materias primas son la semolina y el agua, que luego del amasado son extruidas para darle diferentes formas; sin embargo, como es un alimento que se consume en todo el mundo, es apetecido para la adición de otras materias primas que lo hagan más nutritivo, como es el caso de la incorporación de leguminosas, como sorgo (13), arroz, lenteja, garbanzo (14), amaranto (12) y mijo (15), entre otras. También se han Ilevado a cabo estudios para evaluar el efecto del agua y del gluten en las propiedades fisicoquímicas de pasta "lista para comer" (16). Comparada con otros alimentos con elevada cantidad de carbohidratos, como el pan, la pasta tiene efectos fisiológicos benéficos, como reducir el índice glicémico (17). El objetivo de este trabajo fue elaborar un producto tipo 'pasta' con residuos de plátano hartón prefreído, como 
una alternativa para valorizar estos residuos de la industria de alimentos.

\section{Materiales y métodos}

Reactivos: Los reactivos usados fueron de grado analítico: éter de petróleo Panreac $99 \%$ de pureza, hidróxido de sodio Panreac $99 \%$ de pureza, sulfato de sodio Merck $99 \%$ de pureza, selenio, ácido sulfúrico Panreac $98 \%$ de pureza, ácido bórico Panreac $99 \%$ de pureza y ácido clorhídrico Merck $37 \%$ de pureza, indicador tashiro. Todas las soluciones se prepararon con agua desionizada Sistema Milli-Di ${ }^{\text {TM }}$ y Simplicity con conductividad menor a 0,05 uS.

Equipos: Se verificaron las condiciones de funcionamiento y calibración de los instrumentos usados en los análisis químicos, dentro de los que se encuentran la estufa de secado (Marca MLW), la mufla (Thermolyne F62737), la unidad de destilación (UDK 126D VelpScientifica), el digestor de proteína (DK6 Velp Scientifica) y el pH-metro digital Schott CG 842.

Materiales: El subproducto de patacón (SPP) fue la materia prima principal utilizada; se trata de los recortes que quedan tras el moldeado del patacón, los cuales se almacenaron a temperaturas de refrigeración $\left(4^{\circ} \mathrm{C}\right)$. Se hicieron ensayos con tres marcas comerciales diferentes de harina de trigo (San Agustín, Corona y Haz de Oros) para observar las ventajas y desventajas con respecto a la sémola de trigo, que es la materia prima básica para elaborar pastas alimenticias.

Caracterización del plátano: $\mathrm{Al}$ subproducto de patacón y al plátano hartón se les evaluó la humedad (AOAC 923.03/95. Método directo), la grasa (AOAC 945.16/95. Extracción con éter de petróleo), la proteína (AOAC 984.13. Método de Kjeldahl, N x 6,25) y las cenizas (AOAC 942.05. Método directo) (18).

\section{A. Formulación del producto}

Selección del nivel de inclusión de sémola o harina para la formulación del producto: Se seleccionó el nivel mínimo más adecuado de inclusión de harina de trigo o sémola al subproducto de plátano para elaborar un producto tipo "pasta" (PTP). La elección de las formulaciones estuvo basada en la consistencia obtenida en las pruebas de cocción, el tiempo de almacenamiento del SPP en refrigeración y las características organolépticas del producto final. EI SPP se molió, se mezcló con harina o sémola de trigo y agua (2\%); se amasó y moldeó en una máquina para fabricar pasta italiana casera; se secó a temperatura ambiente y se empacó en bolsas comerciales de polipropileno. Las harinas usadas fueron San Agustín (A), Corona (B), Haz de Oros (C) y sémola de trigo (D). Se elaboraron siete mezclas para establecer el mínimo nivel de inclusión de harina en la formulación del PTP: 1) 60:35, 2) $60: 40$, 3) 55:45, 4) 50:50, 5) 45:55, 6) 40:60 y 7) 35:65 ( $\%$ SSP: \% harina, respectivamente), y todas con $2 \%$ de agua. Para establecer la formulación más adecuada, es decir, el menor nivel de inclusión de harina, para aumentar la utilización de los residuos de plátano hartón prefreído, se evaluaron algunos parámetros de calidad; en primer lugar, el porcentaje de pérdida de agua durante el secado (parámetro: mayor), el cual determina la vida útil del producto, pues una alta disponibilidad de agua en este puede aumentar la carga bacteriana y, por ende, su deterioro; en segundo lugar, el porcentaje de absorción de agua en las pruebas de cocción (mayor porcentaje), pues este es un indicativo de la capacidad de la proteína y del almidón de retener agua y darle al producto la consistencia blanda, y, en tercer lugar, el color del producto tipo "pasta" cocinado y la turbidez del agua de cocción.

Prueba de cocción: Después del amasado y corte del producto tipo "pasta", se secó a temperatura ambiente durante 24 horas y se evaluó el porcentaje de pérdida de agua; posteriormente, se hizo la prueba de cocción, donde se determinó el porcentaje de absorción de agua. En esta fase se evaluó la consistencia del producto cocinado, el tiempo de cocción para las diferentes formas del producto y la turbidez organoléptica del caldo resultante, ya que el alimento se desarrolló con el fin de dar al consumidor una alternativa de preparar sopa de plátano sin que se requiriera acondicionamiento del fruto. 


\section{B. Evaluación de la calidad microbiológica, proximal y sensorial del producto tipo pasta PTP}

Análisis microbiológico: Al producto tipo "pasta alimenticia" se le realizaron los análisis de mesófilos, coliformes fecales, coliformes totales, mohos y levaduras, según la Resolución 4393 de 1991 para Pastas Alimenticias (19).

Análisis proximal: El análisis proximal del PTP se llevó a cabo según los métodos discutidos en la AOAC (18), dentro de los que están grasa (945.16. Extracción con éter de petróleo), humedad (923.03. Método directo), cenizas (942.05. Método directo) y proteína (984.13. Método de Kjeldahl, $\mathrm{N} \times 6,25$ ).

Análisis sensorial: Se determinó una prueba de medición del grado de satisfacción, utilizando una escala hedónica de cinco puntos con un panel no entrenado compuesto por 25 personas, donde se evaluaron aspectos como color, apariencia, aroma, sabor y consistencia del producto tipo "pasta alimenticia", utilizando las cuatro formulaciones elegidas. De acuerdo con la muestra seleccionada, se llevó a cabo un análisis sensorial por el método de puntajes con un panel entrenado compuesto por 9 expertos pertenecientes al Departamento de Química de la Universidad Nacional de Colombia, Sede Bogotá, donde se evaluaron los parámetros nombrados.

\section{Resultados y discusión}

\section{A. Caracterización proximal del plátano}

El plátano hartón verde presentó una humedad de $59,4 \pm 0,3 \%$, grasa de $0,20 \pm 0,3 \%$, proteína de $1,20 \pm 0,2 \%$ y cenizas de $0,8 \pm 0,3 \%$, y el plátano hartón prefreído obtuvo unos resultados de $55,5 \pm 0,2 \%, 9,05 \pm 0,2 \%, 1,42 \pm 0,4 \%$ y $1,18 \pm 0,5 \%$, respectivamente. Se observó que, al ser sometido a un prefreído, el plátano hartón absorbe aceite (de $5 \%$ a $8 \%$ ) y pierde humedad, debido a la transferencia de masa entre el agua y el aceite (20). Dentro de las ventajas de la fritura está la reducción del tiempo de cocción, la mejora de las propiedades organolépticas, haciéndolo más apetecido por el consumidor, y el incremento de la cantidad de vitaminas liposolubles y compuestos bioactivos que provienen de los aceites, como carotenoides, tocoferoles y polifenoles (21). El plátano frito es un snack que se consume ampliamente en Colombia, debido a sus características sensoriales atractivas que incentivan su compra.

\section{B. Formulación del producto}

Determinación del nivel de inclusión de harina: Con el fin de elaborar el producto tipo "Pasta Alimenticia" (PTP) a partir de los residuos de plátano hartón prefreído se determinó el nivel de inclusión de harina. En la Tabla I se observan los resultados obtenidos durante el secado del PTP y se destaca que los más altos porcentajes de pérdida de agua los presenta la formulación 2 (60:40) con la harina de trigo Haz de Oros (C) y con sémola de trigo (D), lo que es beneficioso para la conservación del producto.

Tabla I. Porcentaje de pérdida de agua del PTP durante el secado a temperatura ambiente.

\begin{tabular}{|c|c|c|c|c|}
\hline $\begin{array}{c}\text { HARINA } \\
\text { MEZCLA }\end{array}$ & A & B & C & D \\
\hline 1 & $33,6 \pm 0,6^{* a}$ & $35,6 \pm 0,4^{\mathrm{a}}$ & $38,7 \pm 1,1^{\mathrm{b}}$ & $38,5 \pm 0,3^{\mathrm{b}}$ \\
\hline 2 & $38,0 \pm 0,2^{\mathrm{b}}$ & $38,4 \pm 0,2^{\mathrm{b}}$ & $41,2 \pm 0,6^{\mathrm{b}}$ & $39,5 \pm 0,4^{\mathrm{b}}$ \\
\hline 3 & $36,4 \pm 1,3^{\mathrm{a}}$ & $38,3 \pm 0,8^{\mathrm{b}}$ & $39,1 \pm 0,7^{\mathrm{b}}$ & $38,5 \pm 0,1^{\mathrm{b}}$ \\
\hline 4 & $35,8 \pm 0,5^{\mathrm{a}}$ & $37,1 \pm 1,1^{\mathrm{a}}$ & $36,5 \pm 0,5^{\mathrm{a}}$ & $38,8 \pm 0,4^{\mathrm{b}}$ \\
\hline 5 & $37,2 \pm 0,4^{\mathrm{b}}$ & $39,4 \pm 0,7^{\mathrm{b}}$ & $38,6 \pm 0,4^{\mathrm{b}}$ & $33,7 \pm 0,8^{\mathrm{a}}$ \\
\hline 6 & $34,9 \pm 0,8^{\mathrm{a}}$ & $35,8 \pm 0,3^{\mathrm{a}}$ & $36,3 \pm 0,6^{\mathrm{a}}$ & $34,2 \pm 0,4^{\mathrm{a}}$ \\
\hline 7 & $34,1 \pm 0,3^{\mathrm{a}}$ & $33,4 \pm 0,5^{\mathrm{a}}$ & $34,7 \pm 0,5^{\mathrm{a}}$ & $33,4 \pm 1,1^{\mathrm{a}}$ \\
\hline
\end{tabular}

A: Harina San Agustín, B: Harina Corona, C: Harina Haz de Oros, D: Sémola de trigo. 
*Promedio de las pruebas realizadas por triplicado \pm la desviación estándar.

Diferentes superíndices en una columna dada indican diferencias estadísticamente significativas $(\mathrm{P}<0,05)$.

Formulaciones: 1) 60:35, 2) 60:40, 3) 55:45, 4) $50: 50,5) 45: 55,6)$ 40:60 y 7) 35:65 (\% SSP:\% harina, respectivamente).

Pruebas de cocción: Durante la extrusión y la cocción de la pasta, el almidón se gelatiniza y luego se retrograda, formando una red rígida, mientras que las proteínas se reorganizan e interaccionan con este, mejorando la calidad y las propiedades texturales del producto (22). En la cocción, la formulación 1 del PTP presentó rápida desintegración, dando una turbidez alta al líquido de cocción, lo que indica que el producto no es lo suficientemente estable para soportar el tratamiento, por lo tanto, no se registró la absorción de agua. Se notó que a mayor porcentaje de harina en el producto, mayor funcionalidad del gluten $y$, por ende, se eleva la consistencia y firmeza del producto cocinado (12). El espesor del producto influyó en la absorción de agua en una relación inversamente proporcional. De acuerdo con los resultados que se registran en la Tabla II, la mayor absorción de agua la presentó la mezcla 2 con las harinas B (Corona) y C (Haz de Oros), lo que demuestra la capacidad de absorción y retención de agua de las proteínas de estas harinas, y el propósito de darle una buena consistencia al producto tipo "pasta".

Teniendo en cuenta el tiempo de almacenamiento del "producto tipo pasta" (PTP), se observó que a mayor tiempo de almacenamiento, menor humedad en el (PTP) y, por ende, menor pérdida de humedad por secado; pero luego de la cocción el producto presentó defectos físicos (zonas grises) que pudieron deberse al material de empaque inadecuado, que permite el paso de agua u oxígeno, lo que probablemente favorece las reacciones de pardeamiento o de oxidación de componentes. Se ha evidenciado que la adición de otros ingredientes o agentes reductores disminuyen las características organolépticas de la pasta $(14,12,15,23,13)$, lo que es similar a lo presentado en este estudio.

Tabla II. Porcentaje de absorción de agua del PTP durante las pruebas de cocción teniendo en cuenta que el $100 \%$ es el peso del producto seco.

\begin{tabular}{|c|c|c|c|c|}
\hline $\begin{array}{c}\text { HARINA } \\
\text { MEZCLA }\end{array}$ & A & B & C & D \\
\hline $\mathbf{1}$ & - & - & - & - \\
\hline $\mathbf{2}$ & $305,5 \pm 0,2^{\mathrm{a}}$ & $327,2 \pm 0,2^{\mathrm{b}}$ & $335,1 \pm 0,2^{\mathrm{b}}$ & $286,5 \pm 0,1^{\mathrm{c}}$ \\
\hline $\mathbf{3}$ & $295,5 \pm 0,2^{\mathrm{c}}$ & $275,6 \pm 0,3^{\mathrm{d}}$ & $301,2 \pm 0,3^{\mathrm{a}}$ & $306,4 \pm 0,1^{\mathrm{a}}$ \\
\hline $\mathbf{4}$ & $301,8 \pm 0,3^{\mathrm{a}}$ & $282,8 \pm 0,2^{\mathrm{c}}$ & $309,7 \pm 0,3^{\mathrm{d}}$ & $303,7 \pm 0,2^{\mathrm{a}}$ \\
\hline $\mathbf{5}$ & $270,1 \pm 0,1^{\mathrm{d}}$ & $298,5 \pm 0,1^{\mathrm{c}}$ & $287,2 \pm 0,3^{\mathrm{c}}$ & $247,3 \pm 0,2^{\mathrm{e}}$ \\
\hline $\mathbf{6}$ & $279,2 \pm 0,2^{\mathrm{c}}$ & $275,9 \pm 0,3^{\mathrm{d}}$ & $292,1 \pm 0,2^{\mathrm{c}}$ & $278,6 \pm 0,2^{\mathrm{c}}$ \\
\hline $\mathbf{7}$ & $215,2 \pm 0,2^{\mathrm{f}}$ & $232,6 \pm 0,2^{\mathrm{f}}$ & $250,5 \pm 0,3^{\mathrm{e}}$ & $227,4 \pm 0,3^{\mathrm{f}}$ \\
\hline
\end{tabular}

A: Harina San Agustín, B: Harina Corona, C: Harina Haz de Oros, D: Sémola de trigo.

* Promedio de las pruebas realizadas por triplicado \pm la desviación estándar.

Diferentes superíndices en una columna dada indican diferencias estadísticamente significativas $(P<0,05)$.

Para la selección de la formulación más agradable se eligió, inicialmente, la mezcla 2 con las harinas C y D, por su buena capacidad de secado y absor- ción de agua, y además por su color y la turbidez del líquido de cocción, factor fundamental, ya que el producto se quiere para consumo en sopa; pero su consistencia no fue muy blanda, por lo que se planteó la alternativa de tomar la mezcla 1 con las mismas harinas (mejor color) y darle un espesor 
más grueso para evitar la desintegración del producto durante la cocción; las características sensoriales obtenidas fueron buenas y seleccionaron para, junto con las dos ya elegidas, someterlas a una prueba sensorial con consumidores para que ellos eligieran la formulación que más les agradaba.

Análisis sensorial: Se elaboró una prueba afectiva con consumidores usando una escala hedónica de cinco puntos para que los panelistas seleccionaran el producto tipo "pasta" que más les agradara dentro de las cuatro formulaciones seleccionadas: 1) 65:35 (Muestra 001 Harina C), 2) 65:35 (Muestra
002 Harina D), 3) 60:40 (Muestra 003 Harina C y 4) 60:40 (Muestra 004 Harina D). Además, se evaluó el caldo elaborado con el producto (Tabla III). De acuerdo con los resultados, el PTP elaborado con sémola de trigo fue el más aceptado sensorialmente. Teniendo en cuenta las observaciones anotadas por los panelistas, se concluye que el sabor del caldo depende de la sustancia adicionada como condimento, ya que el producto tipo "pasta" tiene aroma y sabor muy leves. Finalmente, se seleccionó la muestra 002 debido a sus buenas características sensoriales y por su mínimo nivel de inclusión de harina.

Tabla III. Promedio de las características sensoriales para las formulaciones seleccionadas del PTP y el caldo elaborados con estas

\begin{tabular}{|c|c|c|c|c|}
\hline Muestra & Apariencia & Color & Aroma & Sabor \\
\hline \multicolumn{5}{|c|}{ Pasta } \\
\hline 001 & $2,4 \pm 0,2^{* \mathrm{a}}$ & $2,1 \pm 0,4^{\mathrm{a}}$ & $3,4 \pm 0,3^{\mathrm{a}}$ & $3,1 \pm 0,2^{\mathrm{a}}$ \\
\hline 002 & $3,4 \pm 0,3^{\mathrm{b}}$ & $3,2 \pm 0,3^{\mathrm{b}}$ & $4,0 \pm 0,3^{\mathrm{b}}$ & $3,4 \pm 0,2^{\mathrm{a}}$ \\
\hline 003 & $3,0 \pm 0,2^{\mathrm{b}}$ & $2,7 \pm 0,2^{\mathrm{b}}$ & $3,2 \pm 0,2^{\mathrm{a}}$ & $2,4 \pm 0,2^{\mathrm{b}}$ \\
\hline 004 & $3,2 \pm 0,2^{\mathrm{b}}$ & $3,1 \pm 0,3^{\mathrm{b}}$ & $3,5 \pm 0,2^{\mathrm{a}}$ & $3,3 \pm 0,3^{\mathrm{a}}$ \\
\hline \multicolumn{5}{|c|}{ Caldo } \\
\hline 001 & $3,1 \pm 0,2^{\mathrm{b}}$ & $3,2 \pm 0,4^{\mathrm{b}}$ & $4,2 \pm 0,2^{\mathrm{b}}$ & $4,3 \pm 0,1^{\mathrm{c}}$ \\
\hline 002 & $3,9 \pm 0,3^{\mathrm{c}}$ & $3,8 \pm 0,1^{\mathrm{c}}$ & $4,1 \pm 0,3^{\mathrm{b}}$ & $4,2 \pm 0,2^{\mathrm{c}}$ \\
\hline 003 & $3,2 \pm 0,3^{\mathrm{a}}$ & $3,1 \pm 0,2^{\mathrm{b}}$ & $3,5 \pm 0,2^{\mathrm{a}}$ & $3,8 \pm 0,2^{\mathrm{c}}$ \\
\hline 004 & $3,8 \pm 0,5^{\mathrm{c}}$ & $3,6 \pm 0,1^{\mathrm{c}}$ & $4,0 \pm 0,2^{\mathrm{b}}$ & $4,1 \pm 0,1^{\mathrm{c}}$ \\
\hline
\end{tabular}

* Promedio de las pruebas realizadas por triplicado \pm la desviación estándar.

Diferentes superíndices en una columna dada indican diferencias estadísticamente significativas $(\mathrm{P}<0,05)$.

\section{Evaluación de la calidad microbiológica, proxi- mal y sensorial del PTP}

Análisis microbiológico: Los resultados de los análisis microbiológicos Ilevados a cabo al producto seleccionado (Muestra 002: 65:35 sémola de trigo y SPP), comparados con la norma (19), indicaron que este alimento es apto para el consumo humano y que los niveles de microorganismos son inferiores a los máximos permisibles para clasificar la pasta dentro del nivel de buena calidad. El recuento de mesófilos fue menor a $10 \mathrm{ufc/g}$, el nú- mero más probable (NMP) de coliformes totales y fecales/g fue menor de 3 y el conteo de mohos y levaduras fue de $40 \mathrm{ufc/g}$.

Análisis proximal: En la Tabla IV se exponen los resultados del análisis proximal realizado al PTP comparado con una pasta comercial y con la norma vigente para este tipo de alimento. EI PTP tiene mayor cantidad de grasa y la mitad de proteína, comparado con una pasta comercial, lo que se evidencia estadísticamente. Como se esperaba, el contenido de proteína en el PTP es inferior al que se presenta en una pasta comercial y no está dentro del rango mínimo que pide la norma, que es 10,5 $\%$, y aunque la grasa cruda está dentro del rango, es elevada para un producto de este tipo. Actual- 
mente se ha observado un incremento en estudios enfocados en la obtención de productos tipo pasta bajos en gluten y grasa, con la adición de legumbres que aumenten la porción proteica y propor- cionen las características organolépticas propias de la pasta $(24,25$ through an extrusion-cooking process (Brabender $10 \mathrm{DN}$ single-screw extruder with a 3:1 compression ratio,13).

Tabla IV. Análisis proximal del PTP comparado con una pasta comercial y con la norma vigente

\begin{tabular}{|l|c|c|c|}
\hline \multicolumn{1}{|c|}{ Componente } & $\begin{array}{c}\text { Producto tipo } \\
\text { "pasta" }\end{array}$ & Pasta comercial & Norma [27] \\
\hline Humedad (\%) & $12,50 \pm 0,2^{\text {a }}$ & $10,00 \pm 0,3^{\text {a }}$ & Máx. 13,00 \\
\hline Grasa cruda (\%) & $7,02 \pm 0,2^{\text {a }}$ & $2,00 \pm 0,2^{\text {b }}$ & Mín. 0,40 \\
\hline Proteína cruda (\%) & $6,26 \pm 0,05^{\mathrm{a}}$ & $12,00 \pm 0,3^{\mathrm{b}}$ & Mín. 10,5 \\
\hline Cenizas (\%) & $1,38 \pm 0,1^{\mathrm{a}}$ & $1,20 \pm 0,2^{\mathrm{a}}$ & Máx. 0,80 \\
\hline Carbohidratos (\%)* & $73,34^{\mathrm{a}}$ & $74,80^{\mathrm{a}}$ & - \\
\hline Kcal/ $100 \mathrm{~g}$ & $381^{\mathrm{a}}$ & $365^{\mathrm{a}}$ & - \\
\hline
\end{tabular}

* Promedio de las pruebas realizadas por triplicado \pm la desviación estándar. ** Calculados por diferencia

Diferentes superíndices en una fila dada indican diferencias estadísticamente significativas $(P<0,05)$.

Análisis sensorial: En la evaluación de la calidad sensorial, llevada a cabo por el panel entrenado en análisis sensorial, se observó que el PTP sin tratamiento de cocción tiene un color uniforme, similar al plátano hartón verde prefreído, un aroma muy leve a este último y los efectos no afectan la apariencia y calidad del producto. En el producto cocinado el color es similar al SPP, con una consistencia ligeramente dura, harinosa y gomosa. La sopa elaborada no presenta aroma ni sabor a plátano hartón verde prefreído, solo un aroma muy leve a plátano, pues se encuentra enmascarado con la arveja y la zanahoria de la sopa.

Al evaluar la calidad microbiológica, proximal y sensorial se determinó que el producto tipo pasta alimenticia es inocuo para el consumo humano, y aunque no cumple con la norma de pastas alimenticias referente al contenido de proteína y grasa puede ser una opción para su uso en la elaboración de sopas, pues las características sensoriales son aptas para un producto de este tipo.

\section{Conclusión}

Es factible elaborar un producto tipo "pasta" (PTP), con características sensoriales aceptables, a partir de subproducto de plátano hartón prefreído (SPP); la formulación más adecuada para ello es la preparada con una relación de 35:65 de sémola de trigo y SPP. Según los resultados del análisis proximal, el PTP presenta mayor cantidad de grasa y menor contenido de proteína que una pasta comercial. EI PTP puede ser usado como ingrediente en la elaboración de sopas cuyas características sensoriales dependen de otros ingredientes adicionados, ya que el SPP tiene bajas características de aroma y de sabor a plátano hartón freído.

\section{Agradecimientos}

La autora agradece al Grupo de Investigación en Alimentos Funcionales de la Universidad Nacional de Colombia-Sede Bogotá, por el soporte financiero del proyecto, y a la profesora Ana Silvia Bermúdez, por su apoyo y dedicación.

\section{Referencias}

(1) Passo Tsamo CV, Herent MF, Tomekpe K., Happi Emaga T., Quetin-Leclercq JH, Rogez J., Larondelle Y., Andre C. Phenolic profiling in the pulp and peel of nine plantain cultivars (Musa sp.). Food Chem. 2015; 167: 197-204. DOI: http://doi.org/10.1016/j. foodchem.2014.06.095.

(2) Turner DW, Fortescue JA, Ocimati W, Blomme G. Plantain cultivars (Musa spp. AAB) grown at different altitudes demonstrate cool temperature 
and photoperiod responses relevant to genetic improvement. F Crop Res. 2016; 194: 103-111. DOI: http://doi.org/10.1016/j.fcr.2016.02.006.

(3) Olmos Soler AM. Cadena Productiva del Plátano. Ministerio de Agricultura y Desarrollo Rural. 2015.

(4) FAO. Panorama General de la Producción y el Comercio Mundial de Banano. Depósitos de documentos de la FAO. 2010. Disponible en: http:// www.fao.org/docrep/007/y5102s/y5102s04.htm.

(5) Martínez H. La Cadena del Plátano en Colombia. Bogotá (Colombia). 2005.

(6) Pérez M, Ruiz D, Schneider M, Autino J, Romanelli G. La química verde como fuente de nuevos compuestos para el control de plagas agrícolas. Cienc en Desarro. 2013; 4(2): 83-91.

(7) Quaglia G.Ciencia y Tecnología de la Panificación. Zaragoza (España). 1991.

(8) ICBF. Tabla de composición de los Alimentos Colombianos. 2016. Disponible en: http://alimentoscolombianos.icbf.gov.co/alimentos_colombianos/ consulta_alimento.asp.

(9) Wang L., Duan W., Zhou S., Quian H., Zhang H., Qi X. Effects of extrusion conditions on the extrusion responses and the quality of brown rice pasta. Food Chem. 2016; 204: 320-325. DOI: http://doi. org/10.1016/j.foodchem.2016.02.053.

(10) Ficco DBM, De Simone V., Colecchia S., Pecorella I., Platani C., Nigro F., Finocchiaro F., Papa R., De Vita P. Genetic Variability in Anthocyanin Composition and Nutritional Properties of Blue, Purple, and Red Bread (Triticum aestivum L.) and Durum (Triticum turgidum L. ssp. turgidum convar. durum) Wheats. J Agric Food Chem. 2014; 62 (34): 86868695. DOI: http://doi.org/10.1021/jf5003683.

(11) Ficco DBM, De Simone V., De Leonardis AM, Giovanniello V., Del Nobile MA, Padalino L., Lecce L., Borrelli GM, De Vita P. Use of purple durum wheat to produce naturally functional fresh and dry pasta. Food Chem. 2016; 205: 187-195. DOI: http://doi. org/10.1016/j.foodchem.2016.03.014.

(12) Cabrera-Chávez F., Calderón de la Barca AM, Islas-Rubio A. R., Marti A., Marengo M., Pagani MA, Bonomi F., lametti S. Molecular rearrangements in extrusion processes for the production of amaranth-enriched, gluten-free rice pasta. LWT - Food Sci Technol. 2012; 47(2): 421-426. DOI: http://doi. org/10.1016/j.Iwt.2012.01.040.
(13) Marengo M., Bonomi F., Marti A., Pagani MA, Elkhalifa AEO, lametti S. Molecular features of fermented and sprouted sorghum flours relate to their suitability as components of enriched gluten-free pasta. LWT - Food Sci Technol. 2015; 63(1): 511-518. DOI: http://doi.org/10.1016/j. Iwt.2015.03.070.

(14) Bouasla A., Wójtowicz A., Zidoune MN. Gluten-free precooked rice pasta enriched with legumes flours: Physical properties, texture, sensory attributes and microstructure. LWT - Food Sci Technol. 2016; 75: 569-577. DOI: http://doi. org/10.1016/j.lwt.2016.10.005.

(15) Jalgaonkar K, Jha SK. Influence of particle size and blend composition on quality of wheat semolina-pearl millet pasta. J Cereal Sci. 2016; 71: 239-245. DOI: http://doi.org/10.1016/j. jcs.2016.09.007.

(16) Diantom A., Carini E., Curti E., Cassotta F., D'Alessandro A., Vittadini E. Effect of water and gluten on physico-chemical properties and stability of ready to eat shelf-stable pasta. Food Chem. 2016; 195: 91-96. DOI: http://doi.org/10.1016/j.foodchem.2015.04.026.

(17) Aston LM, Gambell JM, Lee DM, Bryant SP, Jebb $\mathrm{SA}$. Determination of the glycaemic index of various staple carbohydrate-rich foods in the UK diet. Eur J Clin Nutr. 2008; 62: 279-285. DOI: http://doi. org/10.1038/sj.ejcn.1602723.

(18) AOAC, Official Methods of Analysis, 16th ed. AOAC INTERNATIONAL. 1998.

(19) Ministerio de Salud. Resolución No. 4393 de 1991. Fabricación, empaque y comercialización de Pastas Alimenticias. 1991.

(20) Bassama J., Achir N., Trystram G., Collignan A., Bohuon P. Deep-fat frying process induces nutritional composition diversity of fried products assessed by SAIN/LIM scores. J Food Eng. 2015; 149: 204-213. DOI: http://doi.org/10.1016/j.jfoodeng.2014.10.017.

(21) Bastida S., Sánchez-Muniz FJ. Frying. A. Cultural Way of Cooking in the Mediterranean Diet. The Mediterranean Diet: An Evidence-Based Approach. 2014; 217-234.

(22) lametti S., Bonomi F., Pagani MA, Zardi M., Cecchini C., D'Egidio MG. Properties of the proteins and carbohydrate fractions in immature wheat ker- 
nels. J Agric Food Chem. 2006; 54: 10239-10244. DOI: http://doi.org/10.1021/jf062269t.

(23) Larrosa V., Lorenzo G., Zaritzky N., Califano A. Improvement of the texture and quality of cooked gluten-free pasta. LWT - Food Sci Technol. 2016; 70: 96-103. DOI: http://doi.org/10.1016/j. Iwt.2016.02.039.

(24) Giuberti G., Gallo A., Cerioli C., Fortunati P., Masoero F. Cooking quality and starch digest- ibility of gluten free pasta using new bean flour. Food Chem. 2015; 175: 43-49. DOI: http://doi. org/10.1016/j.foodchem.2014.11.127.

(25) Giménez MA, González RJ, Wagner J., Torres R., Lobo MO, Samman NC. Effect of extrusion conditions on physicochemical and sensorial properties of corn-broad beans (Vicia faba) spaghetti type pasta. Food Chem. 2013; 136(2): 538-545. DOI: http:// doi.org/10.1016/j.foodchem.2012.08.068. 